\title{
Common fixed point theorems for Lipschitz-type fuzzy mappings in metric spaces
}

\author{
Ming-Liang Song* and Xue-Jun Zhang
}

\section{"Correspondence:}

mlsong2004@163.com

Mathematics and Information

Technology School, Jiangsu Second

Normal University, Nanjing, 210013,

P.R. China

\begin{abstract}
In this paper, some common fixed point theorems for Lipschitz-type fuzzy mappings in complete metric spaces are obtained. As applications, we establish some common fixed point theorems for Lipschitz-type multi-valued mappings in complete metric spaces. Also, we give an example to show the validity of our results, which indicates that our results improve and extend several known results in the existing literature.
\end{abstract}

MSC: $47 \mathrm{H} 10 ; 47 \mathrm{H} 04 ; 26 \mathrm{~A} 16$

Keywords: fuzzy set; fuzzy mapping; fixed point; Lipschitz-type fuzzy mapping

\section{Introduction and preliminaries}

The study of fixed point theorems in fuzzy mathematics was instigated by Weiss [1] and Butnariu [2]. Heilpern [3] introduced the concept of fuzzy contractive mappings and proved a fixed point theorem for these mappings in metric linear spaces. His result is a generalization of the fixed point theorem for point-to-set maps of Nadler [4]. Afterwords several fixed point theorems for fuzzy contractive mappings have appeared in the literature (see [5-14]). Especially, Vijayaraju and Marudai [5], Azam and Arshad [6], Bose [14], Frigona and O'Regan studied some fixed point results for fuzzy (multi-valued) mappings $T: X \rightarrow \mathscr{F}(X)$ in a metric space $X$ respectively. This result is significant as it does not require the condition of approximate quantity for $T(x)$ and linearity for $X$. Recently, Zhang [15] established some new common fixed point theorems for Lipschitz-type mappings in cone metric spaces. These theorems extended the known contractive-type conditions.

The aim of this paper is to investigate some common fixed point theorems for Lipschitztype fuzzy mappings in complete metric spaces. As applications, we establish some common fixed point theorems for Lipschitz-type multi-valued mappings in complete metric spaces. Also, we give an example to show the validity of our results, which indicates that our results improve and extend several known results in the existing literature.

Throughout this paper we shall use the following notations and lemmas which were taken from $[2-6,16,17]$.

Let $X$ and $Y$ be nonempty sets. A multi-valued mapping $T$ from $X$ to $Y$, denoted by $T: X \rightarrow 2^{Y}$, is defined to be a function that assigns to each element of $X$ a nonempty subset of $Y$. Fixed points of the multi-valued mapping $T: X \rightarrow 2^{X}$ will be the points $x \in X$ such that $x \in T(x)$.

○2013 Song and Zhang; licensee Springer. This is an Open Access article distributed under the terms of the Creative Commons Attribution License (http://creativecommons.org/licenses/by/2.0), which permits unrestricted use, distribution, and reproduction in any medium, provided the original work is properly cited. 
Let $(X, d)$ be a metric space, and let $\mathscr{C} \mathscr{B}(X)$ denote the set of all nonempty closed and bounded subsets of $X$. For $A, B \in \mathscr{C} \mathscr{B}(X)$ define

$$
H(A, B)=\max \left\{\sup _{x \in A} d(x, B), \sup _{y \in B} d(A, y)\right\},
$$

where

$$
d(x, A)=\inf _{y \in A} d(x, y) .
$$

A fuzzy set in $X$ is a function with domain $X$ and values in [0,1]. If $A$ is a fuzzy set and $x \in X$, then the function value $A(x)$ is called the grade of membership of $x$ in $A$. The $\alpha$-level set of $A$ is denoted by $[A]_{\alpha}$ and is defined as follows:

$$
\begin{aligned}
& {[A]_{\alpha}=\{x: A(x) \geq \alpha\} \quad \text { if } \alpha \in(0,1],} \\
& {[A]_{0}=\overline{\{x: A(x)>0\}}}
\end{aligned}
$$

Here, $\bar{B}$ denotes the closure of the set $B$. Let $\mathscr{F}(X)$ be the collection of all fuzzy sets in a metric space $X$. For $A, B \in \mathscr{F}(X), A \subset B$ means $A(x) \leq B(x)$ for each $x \in X$.

A mapping $T$ from $X$ to $\mathscr{F}(Y)$ is called a fuzzy mapping if for each $x \in X, T(x)$ (sometimes denoted by $T x$ ) is a fuzzy set on $Y$ and $T x(y)$ denotes the degree of membership of $y$ in $T x$. Let $\mathscr{W}(X)$ denote the set of all fuzzy sets on $X$ such that each of its $\alpha$-level is a nonempty closed bounded subset of $X$.

Lemma 1.1 (Nadler [4]) Let $(X, d)$ be a metric space and $A, B \in \mathscr{C} \mathscr{B}(X)$, then

(1) for each $x \in A, d(x, B) \leq H(A, B)$;

(2) for each $y \in X, d(x, A) \leq d(x, y)+d(y, A)$.

Lemma 1.2 (Nadler [4]) Let $(X, d)$ be a metric space and $A, B \in \mathscr{C} \mathscr{B}(X)$, then for each $x \in A$ and $\varepsilon>0$ there exists an element $y \in B$ such that $d(x, y) \leq H(A, B)+\varepsilon$.

\section{Main results}

In this section, we will establish some common fixed point theorems for a pair of Lipschitztype fuzzy mappings in complete metric spaces.

Theorem 2.1 Let $(X, d)$ be a complete metric space, and let $S, T: X \rightarrow \mathscr{F}(X)$ be two Lipschitz-type fuzzy mappings satisfying the following conditions:

(a) for each $x \in X$, there exists $\alpha(x) \in(0,1]$ such that $[S x]_{\alpha(x)},[T x]_{\alpha(x)}$ are nonempty closed bounded subsets of $X$, and

(b) for all $x, y \in X$,

$$
\begin{aligned}
& H\left([S x]_{\alpha(x)},[T y]_{\alpha(y)}\right) \\
& \quad \leq A_{1}(x, y) d\left(x,[S x]_{\alpha(x)}\right)+A_{2}(x, y) d\left(y,[T y]_{\alpha(y)}\right)+A_{3}(x, y) d\left(x,[T y]_{\alpha(y)}\right) \\
& \quad+A_{4}(x, y) d\left(y,[S x]_{\alpha(x)}\right)+A_{5}(x, y) d(x, y),
\end{aligned}
$$

where $A_{1}, A_{2}, A_{3}, A_{4}, A_{5}$ are five functions from $X \times X$ to $[0,+\infty)$ such that 
(i) $A_{3}(x, y)+A_{4}(x, y)+A_{5}(x, y)<1$ for all $x, y \in X$;

(ii) $\inf _{x, y \in X}\left\{1-A_{1}(x, y)-A_{4}(x, y)\right\}=a, \inf _{x, y \in X}\left\{1-A_{2}(x, y)-A_{3}(x, y)\right\}=b$, $\sup _{x, y \in X}\left\{A_{1}(x, y)+A_{3}(x, y)+A_{5}(x, y)\right\}=A, \sup _{x, y \in X}\left\{A_{2}(x, y)+A_{4}(x, y)+A_{5}(x, y)\right\}=B$, with $a, b, A, B>0$ and $A B<a b$. Then there exists $z \in X$ such that $z \in[S z]_{\alpha(z)} \cap[T z]_{\alpha(z)}$.

Proof Let $x_{0} \in X$. For this $x_{0}$, by condition (a), there exists $\alpha\left(x_{0}\right) \in(0,1]$ such that $\left[S x_{0}\right]_{\alpha\left(x_{0}\right)}$ is a nonempty closed bounded subset of $X$. For convenience, we denote $\alpha\left(x_{0}\right)$ by $\alpha_{1}$. Choose $x_{1} \in\left[S x_{0}\right]_{\alpha_{1}}$, for this $x_{1}$, there exists $\alpha_{2} \in(0,1]$ such that $\left[T x_{1}\right]_{\alpha_{2}}$ is a nonempty closed bounded subset of $X$. Since $1>0$, by Lemma 1.2, there exists $x_{2} \in\left[T x_{1}\right]_{\alpha_{2}}$ such that

$$
d\left(x_{1}, x_{2}\right) \leq H\left(\left[S x_{0}\right]_{\alpha_{1}},\left[T x_{1}\right]_{\alpha_{2}}\right)+1 .
$$

Since $a, b, A, B>0$, by the same argument, we can find $\alpha_{3} \in(0,1]$ and $x_{3} \in\left[S x_{2}\right]_{\alpha_{3}}$ such that

$$
d\left(x_{2}, x_{3}\right) \leq H\left(\left[S x_{2}\right]_{\alpha_{3}},\left[T x_{1}\right]_{\alpha_{2}}\right)+\frac{A B}{a b} .
$$

By induction we produce a sequence $\left\{x_{n}\right\}$ of points of $X$,

$$
x_{2 k+1} \in\left[S x_{2 k}\right]_{\alpha_{2 k+1}}, \quad x_{2 k+2} \in\left[T x_{2 k+1}\right]_{\alpha_{2 k+2}}, \quad k=0,1,2, \ldots
$$

such that

$$
\begin{aligned}
& d\left(x_{2 k+1}, x_{2 k+2}\right) \leq H\left(\left[S x_{2 k}\right]_{\alpha_{2 k+1}},\left[T x_{2 k+1}\right]_{\alpha_{2 k+2}}\right)+\left(\frac{A B}{a b}\right)^{k}, \\
& d\left(x_{2 k+2}, x_{2 k+3}\right) \leq H\left(\left[S x_{2 k+2}\right]_{\alpha_{2 k+3}},\left[T x_{2 k+1}\right]_{\alpha_{2 k+2}}\right)+\left(\frac{A B}{a b}\right)^{k+1} .
\end{aligned}
$$

For $k=0,1,2, \ldots$, applying (2.1), (2.2) and condition (i), we obtain

$$
\begin{aligned}
d\left(x_{2 k+1}, x_{2 k+2}\right) \leq & H\left(\left[S x_{2 k}\right]_{\alpha_{2 k+1}},\left[T x_{2 k+1}\right]_{\alpha_{2 k+2}}\right)+\left(\frac{A B}{a b}\right)^{k} \\
\leq & A_{1}\left(x_{2 k}, x_{2 k+1}\right) d\left(x_{2 k},\left[S x_{2 k}\right]_{\alpha_{2 k+1}}\right) \\
& +A_{2}\left(x_{2 k}, x_{2 k+1}\right) d\left(x_{2 k+1},\left[T x_{2 k+1}\right]_{\alpha_{2 k+2}}\right) \\
& +A_{3}\left(x_{2 k}, x_{2 k+1}\right) d\left(x_{2 k},\left[T x_{2 k+1}\right]_{\alpha_{2 k+2}}\right) \\
& +A_{4}\left(x_{2 k}, x_{2 k+1}\right) d\left(x_{2 k+1},\left[S x_{2 k}\right]_{\alpha_{2 k+1}}\right) \\
& +A_{5}\left(x_{2 k}, x_{2 k+1}\right) d\left(x_{2 k}, x_{2 k+1}\right)+\left(\frac{A B}{a b}\right)^{k} \\
\leq & A_{1}\left(x_{2 k}, x_{2 k+1}\right) d\left(x_{2 k}, x_{2 k+1}\right)+A_{2}\left(x_{2 k}, x_{2 k+1}\right) d\left(x_{2 k+1}, x_{2 k+2}\right) \\
& +A_{3}\left(x_{2 k}, x_{2 k+1}\right) d\left(x_{2 k}, x_{2 k+2}\right)+A_{4}\left(x_{2 k}, x_{2 k+1}\right) d\left(x_{2 k+1}, x_{2 k+1}\right) \\
& +A_{5}\left(x_{2 k}, x_{2 k+1}\right) d\left(x_{2 k}, x_{2 k+1}\right)+\left(\frac{A B}{a b}\right)^{k} \\
\leq & A_{1}\left(x_{2 k}, x_{2 k+1}\right) d\left(x_{2 k}, x_{2 k+1}\right)+A_{2}\left(x_{2 k}, x_{2 k+1}\right) d\left(x_{2 k+1}, x_{2 k+2}\right)
\end{aligned}
$$




$$
\begin{aligned}
& +A_{3}\left(x_{2 k}, x_{2 k+1}\right) d\left(x_{2 k}, x_{2 k+1}\right)+A_{3}\left(x_{2 k}, x_{2 k+1}\right) d\left(x_{2 k+1}, x_{2 k+2}\right) \\
& +A_{5}\left(x_{2 k}, x_{2 k+1}\right) d\left(x_{2 k}, x_{2 k+1}\right)+\left(\frac{A B}{a b}\right)^{k} .
\end{aligned}
$$

It implies that

$$
\begin{aligned}
d\left(x_{2 k+1}, x_{2 k+2}\right) \leq & \frac{A_{1}\left(x_{2 k}, x_{2 k+1}\right)+A_{3}\left(x_{2 k}, x_{2 k+1}\right)+A_{5}\left(x_{2 k}, x_{2 k+1}\right)}{1-A_{2}\left(x_{2 k}, x_{2 k+1}\right)-A_{3}\left(x_{2 k}, x_{2 k+1}\right)} d\left(x_{2 k}, x_{2 k+1}\right) \\
& +\left(\frac{A B}{a b}\right)^{k} \frac{1}{1-A_{2}\left(x_{2 k}, x_{2 k+1}\right)-A_{3}\left(x_{2 k}, x_{2 k+1}\right)} \\
\leq & \frac{\sup _{x, y \in X}\left\{A_{1}(x, y)+A_{3}(x, y)+A_{5}(x, y)\right\}}{\inf _{x, y \in X}\left\{1-A_{2}(x, y)-A_{3}(x, y)\right\}} d\left(x_{2 k}, x_{2 k+1}\right) \\
& +\left(\frac{A B}{a b}\right)^{k} \frac{1}{\inf _{x, y \in X}\left\{1-A_{2}(x, y)-A_{3}(x, y)\right\}} .
\end{aligned}
$$

Note that by condition (ii), we have

$$
d\left(x_{2 k+1}, x_{2 k+2}\right) \leq \frac{A}{b} d\left(x_{2 k}, x_{2 k+1}\right)+\frac{1}{b}\left(\frac{A B}{a b}\right)^{k} .
$$

Similarly, we have

$$
d\left(x_{2 k+2}, x_{2 k+3}\right) \leq \frac{B}{a} d\left(x_{2 k+1}, x_{2 k+2}\right)+\frac{1}{a}\left(\frac{A B}{a b}\right)^{k+1} .
$$

Using the inductive method, for $k=0,1,2, \ldots$, by (2.3) and (2.4), we can obtain

$$
\begin{aligned}
d\left(x_{2 k+1}, x_{2 k+2}\right) \leq & \frac{A}{b} d\left(x_{2 k}, x_{2 k+1}\right)+\frac{1}{b}\left(\frac{A B}{a b}\right)^{k} \\
\leq & \frac{A B}{a b} d\left(x_{2 k-1}, x_{2 k}\right)+\frac{A}{a b}\left(\frac{A B}{a b}\right)^{k}+\frac{1}{b}\left(\frac{A B}{a b}\right)^{k} \\
\leq & \frac{A B}{a b} \cdot \frac{A}{b} d\left(x_{2 k-2}, x_{2 k-1}\right)+\frac{1}{b}\left(\frac{A B}{a b}\right)^{k} \\
& +\frac{A}{a b}\left(\frac{A B}{a b}\right)^{k}+\frac{1}{b}\left(\frac{A B}{a b}\right)^{k} \\
\leq & \left(\frac{A B}{a b}\right)^{2} d\left(x_{2 k-3}, x_{2 k-2}\right)+2\left(\frac{1}{b}+\frac{A}{a b}\right)\left(\frac{A B}{a b}\right)^{k} \\
\leq & \cdots \leq\left(\frac{A B}{a b}\right)^{k} d\left(x_{1}, x_{2}\right)+k\left(\frac{1}{b}+\frac{A}{a b}\right)\left(\frac{A B}{a b}\right)^{k} \\
\leq & \left(\frac{A B}{a b}\right)^{k} \cdot \frac{A}{b} d\left(x_{0}, x_{1}\right)+\frac{1}{b}\left(\frac{A B}{a b}\right)^{k}+k\left(\frac{1}{b}+\frac{A}{a b}\right)\left(\frac{A B}{a b}\right)^{k} \\
\leq & \left(\frac{A B}{a b}\right)^{k} \cdot \frac{A}{b} d\left(x_{0}, x_{1}\right)+(k+1)\left(\frac{1}{b}+\frac{A}{a b}\right)\left(\frac{A B}{a b}\right)^{k}
\end{aligned}
$$


and

$$
\begin{aligned}
d\left(x_{2 k+2}, x_{2 k+3}\right) & \leq \frac{B}{a} \cdot\left(\left(\frac{A B}{a b}\right)^{k} \cdot \frac{A}{b} d\left(x_{0}, x_{1}\right)+(k+1)\left(\frac{1}{b}+\frac{A}{a b}\right)\left(\frac{A B}{a b}\right)^{k}\right)+\frac{1}{a}\left(\frac{A B}{a b}\right)^{k+1} \\
& =\left(\frac{A B}{a b}\right)^{k+1} d\left(x_{0}, x_{1}\right)+\frac{B}{a}(k+1)\left(\frac{1}{b}+\frac{A}{a b}\right)\left(\frac{A B}{a b}\right)^{k}+\frac{1}{a}\left(\frac{A B}{a b}\right)^{k+1} \\
& =\left(\frac{A B}{a b}\right)^{k+1} d\left(x_{0}, x_{1}\right)+(k+1)\left(\frac{1}{A}+\frac{1}{a}\right)\left(\frac{A B}{a b}\right)^{k+1}+\frac{1}{a}\left(\frac{A B}{a b}\right)^{k+1} \\
& \leq\left(\frac{A B}{a b}\right)^{k+1} d\left(x_{0}, x_{1}\right)+(k+2)\left(\frac{1}{A}+\frac{1}{a}\right)\left(\frac{A B}{a b}\right)^{k+1} .
\end{aligned}
$$

Obviously, taking $M=\max \left\{\frac{1}{b}+\frac{A}{a b}, \frac{1}{A}+\frac{1}{a}\right\}$, we have

$$
d\left(x_{2 k+1}, x_{2 k+2}\right) \leq\left(\frac{A B}{a b}\right)^{k} \cdot \frac{A}{b} d\left(x_{0}, x_{1}\right)+M(k+1)\left(\frac{A B}{a b}\right)^{k}
$$

and

$$
d\left(x_{2 k+2}, x_{2 k+3}\right) \leq\left(\frac{A B}{a b}\right)^{k+1} d\left(x_{0}, x_{1}\right)+M(k+2)\left(\frac{A B}{a b}\right)^{k+1} .
$$

Next, we prove that the sequence $\left\{x_{n}\right\}$ is a Cauchy sequence in $X$. For any $k<p$, it follows from (2.5) and (2.6) that

$$
\begin{aligned}
d\left(x_{2 k+1}, x_{2 p+1}\right) \leq & d\left(x_{2 k+1}, x_{2 k+2}\right)+\cdots+d\left(x_{2 p}, x_{2 p+1}\right) \\
\leq & \left(\frac{A}{b} \cdot \sum_{i=k}^{p-1}\left(\frac{A B}{a b}\right)^{i}+\sum_{i=k+1}^{p}\left(\frac{A B}{a b}\right)^{i}\right) d\left(x_{0}, x_{1}\right) \\
& +\left(M \cdot \sum_{i=k}^{p-1}(i+1)\left(\frac{A B}{a b}\right)^{i}+M \cdot \sum_{i=k+1}^{p}(i+1)\left(\frac{A B}{a b}\right)^{i}\right) \\
\leq & \left(\frac{A}{b}+1\right) d\left(x_{0}, x_{1}\right) \cdot \sum_{i=k}^{\infty}\left(\frac{A B}{a b}\right)^{i}+2 M \cdot \sum_{i=k}^{\infty}(i+1)\left(\frac{A B}{a b}\right)^{i} .
\end{aligned}
$$

By a similar reasoning process, we can obtain

$$
\begin{aligned}
& d\left(x_{2 k}, x_{2 p+1}\right) \leq\left(\frac{A}{b}+1\right) d\left(x_{0}, x_{1}\right) \cdot \sum_{i=k}^{\infty}\left(\frac{A B}{a b}\right)^{i}+2 M \cdot \sum_{i=k}^{\infty}(i+1)\left(\frac{A B}{a b}\right)^{i}, \\
& d\left(x_{2 k}, x_{2 p}\right) \leq\left(\frac{A}{b}+1\right) d\left(x_{0}, x_{1}\right) \cdot \sum_{i=k}^{\infty}\left(\frac{A B}{a b}\right)^{i}+2 M \cdot \sum_{i=k}^{\infty}(i+1)\left(\frac{A B}{a b}\right)^{i}, \\
& d\left(x_{2 k+1}, x_{2 p}\right) \leq\left(\frac{A}{b}+1\right) d\left(x_{0}, x_{1}\right) \cdot \sum_{i=k}^{\infty}\left(\frac{A B}{a b}\right)^{i}+2 M \cdot \sum_{i=k}^{\infty}(i+1)\left(\frac{A B}{a b}\right)^{i} .
\end{aligned}
$$

Then there exists $k$ with $\frac{n-1}{2} \leq k \leq \frac{n}{2}$, for any $0<n<m$, such that

$$
d\left(x_{m}, x_{n}\right) \leq\left(\frac{A}{b}+1\right) d\left(x_{0}, x_{1}\right) \cdot \sum_{i=k}^{\infty}\left(\frac{A B}{a b}\right)^{i}+2 M \cdot \sum_{i=k}^{\infty}(i+1)\left(\frac{A B}{a b}\right)^{i} .
$$


Since $a, b, A, B>0$ and $A B<a b$, i.e., $0<\frac{A B}{a b}<1$, it follows from Cauchy's root test that $\sum\left(\frac{A B}{a b}\right)^{n}$ and $\sum(n+1)\left(\frac{A B}{a b}\right)^{n}$ are convergent and hence $\left\{x_{n}\right\}$ is a Cauchy sequence in $X$. Since $X$ is a complete metric space, then there exists $z \in X$ such that $x_{n} \rightarrow z$ as $n \rightarrow \infty$. Without loss of generality, let us assume that $n$ is even. Then by (2.1), (2.2) and Lemma 1.1, we have

$$
\begin{aligned}
d\left(z,[T(z)]_{\alpha(z)}\right) \leq & d\left(z, x_{2 n+1}\right)+d\left(x_{2 n+1},[T(z)]_{\alpha(z)}\right) \\
\leq & d\left(z, x_{2 n+1}\right)+H\left(\left[S x_{2 n}\right]_{\alpha_{2 n+1}},[T(z)]_{\alpha(z)}\right) \\
\leq & d\left(z, x_{2 n+1}\right)+A_{1}\left(x_{2 n}, z\right) d\left(x_{2 n},\left[S x_{2 n}\right]_{\alpha_{2 n+1}}\right) \\
& +A_{2}\left(x_{2 n}, z\right) d\left(z,[T(z)]_{\alpha(z)}\right)+A_{3}\left(x_{2 n}, z\right) d\left(x_{2 n},[T(z)]_{\alpha(z)}\right) \\
& +A_{4}\left(x_{2 n}, z\right) d\left(z,\left[S x_{2 n}\right]_{\alpha_{2 n+1}}\right)+A_{5}\left(x_{2 n}, z\right) d\left(x_{2 n}, z\right) \\
\leq & d\left(z, x_{2 n+1}\right)+A_{1}\left(x_{2 n}, z\right) d\left(x_{2 n}, x_{2 n+1}\right) \\
& +A_{2}\left(x_{2 n}, z\right) d\left(z,[T(z)]_{\alpha(z)}\right)+A_{3}\left(x_{2 n}, z\right) d\left(x_{2 n}, z\right) \\
& +A_{3}\left(x_{2 n}, z\right) d\left(z,[T(z)]_{\alpha(z)}\right) \\
& +A_{4}\left(x_{2 n}, z\right) d\left(z, x_{2 n+1}\right)+A_{5}\left(x_{2 n}, z\right) d\left(x_{2 n}, z\right) .
\end{aligned}
$$

It implies that

$$
\begin{aligned}
(1- & \left.A_{2}\left(x_{2 n}, z\right)-A_{3}\left(x_{2 n}, z\right)\right) d\left(z,[T(z)]_{\alpha(z)}\right) \\
\leq & d\left(z, x_{2 n+1}\right)+A_{1}\left(x_{2 n}, z\right) d\left(x_{2 n}, x_{2 n+1}\right)+A_{3}\left(x_{2 n}, z\right) d\left(x_{2 n}, z\right) \\
\quad & +A_{4}\left(x_{2 n}, z\right) d\left(z, x_{2 n+1}\right)+A_{5}\left(x_{2 n}, z\right) d\left(x_{2 n}, z\right) .
\end{aligned}
$$

Note that $A_{3}(x, y)+A_{4}(x, y)+A_{5}(x, y)<1$ for all $x, y \in X, \inf _{x, y \in X}\left\{1-A_{2}(x, y)-A_{3}(x, y)\right\}=$ $b>0$ and $\sup _{x, y \in X}\left\{A_{1}(x, y)+A_{3}(x, y)+A_{5}(x, y)\right\}=A$, we have

$$
b d\left(z,[T(z)]_{\alpha(z)}\right) \leq d\left(z, x_{2 n+1}\right)+A d\left(x_{2 n}, x_{2 n+1}\right)+d\left(x_{2 n}, z\right)+d\left(z, x_{2 n+1}\right)+d\left(x_{2 n}, z\right),
$$

and hence $d\left(z,[T(z)]_{\alpha(z)}\right) \rightarrow 0$ as $n \rightarrow \infty$. Thus $z \in[T z]_{\alpha(z)}$.

Similarly, we can prove that $z \in[S z]_{\alpha(z)}$. Hence $z \in[S z]_{\alpha(z)} \cap[T z]_{\alpha(z)}$. This completes the proof.

Next, we establish a fuzzy version of Kannan-Reich-type theorem (see [18-20]).

Theorem 2.2 Let $(X, d)$ be a complete metric space, and let $S, T: X \rightarrow \mathscr{F}(X)$ be two Kannan-Reich-type fuzzy mappings satisfying the following conditions:

(a) for each $x \in X$, there exists $\alpha(x) \in(0,1]$ such that $[S x]_{\alpha(x)},[T x]_{\alpha(x)}$ are nonempty closed bounded subsets of $X$, and

(b) for all $x, y \in X$,

$$
\begin{aligned}
H\left([S x]_{\alpha(x)},[T y]_{\alpha(y)}\right) \leq & \beta_{1}(x, y) d\left(x,[S x]_{\alpha(x)}\right) \\
& +\beta_{2}(x, y) d\left(y,[T y]_{\alpha(y)}\right)+\beta_{3}(x, y) d(x, y),
\end{aligned}
$$

where $\beta_{1}, \beta_{2}, \beta_{3}$ are three functions from $X \times X$ to $[0,+\infty)$ such that 
(i) $\beta_{3}(x, y)<1$ for all $x, y \in X$;

(ii) $\sup _{x, y \in X}\left\{\beta_{1}(x, y)\right\}=\gamma_{1}, \sup _{x, y \in X}\left\{\beta_{2}(x, y)\right\}=\gamma_{2}, \sup _{x, y \in X}\left\{\beta_{1}(x, y)+\beta_{3}(x, y)\right\}=A$, $\sup _{x, y \in X}\left\{\beta_{2}(x, y)+\beta_{3}(x, y)\right\}=B$,

with $\gamma_{1}<1, \gamma_{2}<1, A, B>0$ and $A B<\left(1-\gamma_{1}\right)\left(1-\gamma_{2}\right)$. Then there exists $z \in X$ such that $z \in[S z]_{\alpha(z)} \cap[T z]_{\alpha(z)}$.

Proof Let $A_{1}(x, y)=\beta_{1}(x, y), A_{2}(x, y)=\beta_{2}(x, y), A_{3}(x, y) \equiv 0, A_{4}(x, y) \equiv 0, A_{5}(x, y)=\beta_{3}(x, y)$ for all $x, y \in X$, then we have

$$
A_{3}(x, y)+A_{4}(x, y)+A_{5}(x, y)=\beta_{3}(x, y)<1 \quad \text { for all } x, y \in X,
$$

and

$$
\begin{aligned}
& \inf _{x, y \in X}\left\{1-A_{1}(x, y)-A_{4}(x, y)\right\}=1-\sup _{x, y \in X}\left\{\beta_{1}(x, y)\right\}=1-\gamma_{1}, \\
& \inf _{x, y \in X}\left\{1-A_{2}(x, y)-A_{3}(x, y)\right\}=1-\sup _{x, y \in X}\left\{\beta_{2}(x, y)\right\}=1-\gamma_{2}, \\
& \sup _{x, y \in X}\left\{A_{1}(x, y)+A_{3}(x, y)+A_{5}(x, y)\right\}=\sup _{x, y \in X}\left\{\beta_{1}(x, y)+\beta_{3}(x, y)\right\}=A, \\
& \sup _{x, y \in X}\left\{A_{2}(x, y)+A_{4}(x, y)+A_{5}(x, y)\right\}=\sup _{x, y \in X}\left\{\beta_{2}(x, y)+\beta_{3}(x, y)\right\}=B,
\end{aligned}
$$

with $1-\gamma_{1}, 1-\gamma_{2}, A, B>0$ and $A B<\left(1-\gamma_{1}\right)\left(1-\gamma_{2}\right)$, which imply the conditions of Theorem 2.1 are satisfied. Therefore, by Theorem 2.1, Theorem 2.2 is proved.

Remark 2.1 Since each nonlinear contraction includes the case of linear contraction as its special case, each fixed point theorem in the above theorem implies a fixed point theorem for linear contraction. From Theorem 2.1 we obtain the following corollary.

Corollary 2.1 Let $(X, d)$ be a complete metric space. Let $S, T: X \rightarrow \mathscr{F}(X)$ be two fuzzy mappings. Suppose that for each $x \in X$, there exists $\alpha(x) \in(0,1]$ such that $[S x]_{\alpha(x)},[T x]_{\alpha(x)}$ are nonempty closed bounded subsets of $X$ and

$$
\begin{aligned}
H\left([S x]_{\alpha(x)},[T y]_{\alpha(y)}\right) \leq & a_{1} d\left(x,[S x]_{\alpha(x)}\right)+a_{2} d\left(y,[T y]_{\alpha(y)}\right) \\
& +a_{3} d\left(x,[T y]_{\alpha(y)}\right)+a_{4} d\left(y,[S x]_{\alpha(x)}\right)+a_{5} d(x, y),
\end{aligned}
$$

for all $x, y \in X$, where $a_{1}, a_{2}, a_{3}, a_{4}$ are non-negative real numbers $a_{5}>0$ and $\eta>0$ with $\sum_{i=1}^{5} a_{i}=1+\eta, a_{3}+a_{4}+a_{5}<1, a_{1}+a_{4}<1, a_{2}+a_{3}<1$ and $\left(a_{1}-a_{2}\right)\left(a_{3}-a_{4}\right)>2 \eta$. Then there exists $z \in X$ such that $z \in[S z]_{\alpha(z)} \cap[T z]_{\alpha(z)}$.

Proof Let $A_{1}(x, y)=a_{1}, A_{2}(x, y)=a_{2}, A_{3}(x, y)=a_{3}, A_{4}(x, y)=a_{4}, A_{5}(x, y)=a_{5}$ for all $x, y \in X$. It is evident that $0<a_{3}+a_{4}+a_{5}<1,0<1-a_{1}-a_{4}, 0<1-a_{2}-a_{3}, 0<a_{1}+a_{3}+a_{5}$ and $0<a_{2}+a_{4}+a_{5}$.

In addition, note that $a_{5}<1$ and $\left(a_{1}-a_{2}\right)\left(a_{3}-a_{4}\right)>2 \eta$, we have

$$
a_{5}(1+\eta)+a_{1} a_{4}+a_{2} a_{3}<a_{5}+\eta+a_{1} a_{4}+a_{2} a_{3}<a_{5}-\eta+a_{1} a_{3}+a_{2} a_{4} .
$$


Since $\sum_{i=1}^{5} a_{i}=1+\eta$, we can obtain

$$
a_{5}\left(\sum_{i=1}^{5} a_{i}\right)+a_{1} a_{4}+a_{2} a_{3}+a_{1} a_{2}+a_{3} a_{4}<1-a_{1}-a_{2}-a_{3}-a_{4}+a_{1} a_{3}+a_{2} a_{4}+a_{1} a_{2}+a_{3} a_{4},
$$

i.e., $\left(a_{1}+a_{3}+a_{5}\right)\left(a_{2}+a_{4}+a_{5}\right)<\left(1-a_{1}-a_{4}\right)\left(1-a_{2}-a_{3}\right)$. Then we easily see that conditions (i) and (ii) of Theorem 2.1 are satisfied. Therefore, by Theorem 2.1, Corollary 2.1 is proved.

Applying Theorem 2.1, we easily obtain the following fixed point theorem for Bose-type fuzzy mappings.

Theorem 2.3 (Bose [14]) Let $(X, d)$ be a complete metric space. Let $S, T: X \rightarrow \mathscr{F}(X)$ be two fuzzy mappings. Suppose that for each $x \in X$, there exists $\alpha(x) \in(0,1]$ such that $[S x]_{\alpha(x)}$, $[T X]_{\alpha(x)}$ are nonempty closed bounded subsets of $X$ and

$$
\begin{aligned}
H\left([S x]_{\alpha(x)},[T y]_{\alpha(y)}\right) \leq & b_{1} d\left(x,[S x]_{\alpha(x)}\right)+b_{2} d\left(y,[T y]_{\alpha(y)}\right) \\
& +b_{3} d\left(y,[S x]_{\alpha(x)}\right)+b_{4} d\left(x,[T y]_{\alpha(y)}\right)+b_{5} d(x, y),
\end{aligned}
$$

for all $x, y \in X$, where $b_{1}, b_{2}, b_{3}, b_{4}, b_{5}$ are non-negative real numbers and $\sum_{i=1}^{5} b_{i}<1$ and $b_{1}=b_{2}$ or $b_{3}=b_{4}$. Then there exists $z \in X$ such that $z \in[S z]_{\alpha(z)} \cap[T z]_{\alpha(z)}$.

Proof If $b_{1}=b_{2}$ and $b_{3}=b_{4}$, we can take $3 \delta=1-\left(\sum_{i=1}^{5} b_{i}\right)>0$ and let $A_{1}(x, y)=b_{1}+\delta$, $A_{2}(x, y)=b_{2}, A_{3}(x, y)=b_{4}+\delta, A_{4}(x, y)=b_{3}, A_{5}(x, y)=b_{5}+\delta$ for all $x, y \in X$, then we have

$$
A_{3}(x, y)+A_{4}(x, y)+A_{5}(x, y)=b_{3}+b_{4}+b_{5}+2 \delta<1 \quad \text { for all } x, y \in X \text {, }
$$

and

$$
\begin{aligned}
& \inf _{x, y \in X}\left\{1-A_{1}(x, y)-A_{4}(x, y)\right\}=1-b_{1}-\delta-b_{3}>0, \\
& \inf _{x, y \in X}\left\{1-A_{2}(x, y)-A_{3}(x, y)\right\}=1-b_{2}-b_{4}-\delta>0, \\
& \sup _{x, y \in X}\left\{A_{1}(x, y)+A_{3}(x, y)+A_{5}(x, y)\right\}=b_{1}+b_{4}+b_{5}+3 \delta>0, \\
& \sup _{x, y \in X}\left\{A_{2}(x, y)+A_{4}(x, y)+A_{5}(x, y)\right\}=b_{2}+b_{3}+b_{5}+\delta>0 .
\end{aligned}
$$

Note that $\delta>0, b_{1}=b_{2}$ and $b_{3}=b_{4}$, it is not difficult to see that

$$
\begin{aligned}
\left(b_{1}+b_{4}+b_{5}+3 \delta\right)\left(b_{2}+b_{3}+b_{5}+\delta\right) & =\left(b_{1}+b_{3}+b_{5}+3 \delta\right)\left(b_{1}+b_{3}+b_{5}+\delta\right) \\
& <\left(b_{1}+b_{3}+b_{5}+2 \delta\right)\left(b_{1}+b_{3}+b_{5}+2 \delta\right) \\
& =\left(b_{2}+b_{4}+b_{5}+2 \delta\right)\left(b_{1}+b_{3}+b_{5}+2 \delta\right) \\
& =\left(1-b_{1}-\delta-b_{3}\right)\left(1-b_{2}-b_{4}-\delta\right) .
\end{aligned}
$$

Then we know that conditions (i) and (ii) of Theorem 2.1 are satisfied. 
In addition, it is evident that

$$
\begin{aligned}
H\left([S x]_{\alpha(x)},[T y]_{\alpha(y)}\right) \leq & b_{1} d\left(x,[S x]_{\alpha(x)}\right)+b_{2} d\left(y,[T y]_{\alpha(y)}\right) \\
& +b_{3} d\left(y,[S x]_{\alpha(x)}\right)+b_{4} d\left(x,[T y]_{\alpha(y)}\right)+b_{5} d(x, y) \\
\leq & \left(b_{1}+\delta\right) d\left(x,[S x]_{\alpha(x)}\right)+b_{2} d\left(y,[T y]_{\alpha(y)}\right) \\
& +\left(b_{4}+\delta\right) d\left(x,[T y]_{\alpha(y)}\right)+b_{3} d\left(y,[S x]_{\alpha(x)}\right)+\left(b_{5}+\delta\right) d(x, y)
\end{aligned}
$$

for all $x, y \in X$, which satisfies inequality (2.1) of Theorem 2.1. Therefore, by Theorem 2.1, the conclusion of Theorem 2.3 holds.

If $b_{1}=b_{2}$ and $b_{3}>b_{4}$, we can take $2 \delta=1-\left(\sum_{i=1}^{5} b_{i}\right)>0$ and let $A_{1}(x, y)=b_{1}, A_{2}(x, y)=$ $b_{2}+\delta, A_{3}(x, y)=b_{4}, A_{4}(x, y)=b_{3}, A_{5}(x, y)=b_{5}+\delta$ for all $x, y \in X$, then we have

$$
A_{3}(x, y)+A_{4}(x, y)+A_{5}(x, y)=b_{3}+b_{4}+b_{5}+\delta<1 \quad \text { for all } x, y \in X
$$

and

$$
\begin{aligned}
& \inf _{x, y \in X}\left\{1-A_{1}(x, y)-A_{4}(x, y)\right\}=1-b_{1}-b_{3}>0, \\
& \inf _{x, y \in X}\left\{1-A_{2}(x, y)-A_{3}(x, y)\right\}=1-b_{2}-b_{4}-\delta>0, \\
& \sup _{x, y \in X}\left\{A_{1}(x, y)+A_{3}(x, y)+A_{5}(x, y)\right\}=b_{1}+b_{4}+b_{5}+\delta>0, \\
& \sup _{x, y \in X}\left\{A_{2}(x, y)+A_{4}(x, y)+A_{5}(x, y)\right\}=b_{2}+b_{3}+b_{5}+2 \delta>0 .
\end{aligned}
$$

Note that $\delta>0, b_{1}=b_{2}$ and $b_{3}>b_{4}$, it is not difficult to see that

$$
\begin{aligned}
\left(b_{1}+b_{4}+b_{5}+\delta\right)\left(b_{2}+b_{3}+b_{5}+2 \delta\right) & =\left(b_{1}+b_{4}+b_{5}+\delta\right)\left(b_{1}+b_{3}+b_{5}+2 \delta\right) \\
& <\left(b_{1}+b_{4}+b_{5}+2 \delta\right)\left(b_{1}+b_{3}+b_{5}+\delta\right) \\
& =\left(1-b_{1}-b_{3}\right)\left(1-b_{2}-b_{4}-\delta\right) .
\end{aligned}
$$

Then we know that conditions (i) and (ii) of Theorem 2.1 are satisfied.

In addition, it is evident that

$$
\begin{aligned}
H\left([S x]_{\alpha(x)},[T y]_{\alpha(y)}\right) \leq & b_{1} d\left(x,[S x]_{\alpha(x)}\right)+b_{2} d\left(y,[T y]_{\alpha(y)}\right) \\
& +b_{3} d\left(y,[S x]_{\alpha(x)}\right)+b_{4} d\left(x,[T y]_{\alpha(y)}\right)+b_{5} d(x, y) \\
\leq & b_{1} d\left(x,[S x]_{\alpha(x)}\right)+\left(b_{2}+\delta\right) d\left(y,[T y]_{\alpha(y)}\right) \\
& +b_{4} d\left(x,[T y]_{\alpha(y)}\right)+b_{3} d\left(y,[S x]_{\alpha(x)}\right)+\left(b_{5}+\delta\right) d(x, y)
\end{aligned}
$$

for all $x, y \in X$, which satisfies inequality (2.1) of Theorem 2.1. Therefore, by Theorem 2.1, the conclusion of Theorem 2.3 holds.

Similarly, we can prove some cases of $b_{1}=b_{2}, b_{3}<b_{4}$ or $b_{1}>b_{2}, b_{3}=b_{4}$ or $b_{1}<b_{2}, b_{3}=b_{4}$, respectively. Then by Theorem 2.1 , the theorem is proved. 
Note that by the conditions of Theorem 2.3, we can obtain the following fixed point theorem for Vijayaraju-Marudai-type fuzzy mappings.

Corollary 2.2 (Vijayaraju and Marudai [5], Azam and Beg [21]) Let $(X, d)$ be a complete metric space. Let $S, T: X \rightarrow \mathscr{F}(X)$ be two fuzzy mappings. Suppose that for each $x \in X$, there exists $\alpha(x) \in(0,1]$ such that $[S x]_{\alpha(x)},[T x]_{\alpha(x)}$ are nonempty closed bounded subsets of $X$ and

$$
\begin{aligned}
H\left([S x]_{\alpha(x)},[T y]_{\alpha(y)}\right) \leq & b_{1} d\left(x,[S x]_{\alpha(x)}\right)+b_{2} d\left(y,[T y]_{\alpha(y)}\right) \\
& +b_{3} d\left(y,[S x]_{\alpha(x)}\right)+b_{4} d\left(x,[T y]_{\alpha(y)}\right)+b_{5} d(x, y),
\end{aligned}
$$

for all $x, y \in X$, where $b_{1}, b_{2}, b_{3}, b_{4}, b_{5}$ are non-negative real numbers and $\sum_{i=1}^{5} b_{i}<1$ and either $b_{1}=b_{2}$ or $b_{3}=b_{4}$. Then there exists $z \in X$ such that $z \in[S z]_{\alpha(z)} \cap[T z]_{\alpha(z)}$.

In Corollary 2.2, if $b_{3}=b_{4}$, then we can obtain the following fixed point theorem for Azam-Arshad-type fuzzy mappings.

Corollary 2.3 (Azam and Arshad [6]) Let $(X, d)$ be a complete metric space. Let $S, T: X \rightarrow$ $\mathscr{F}(X)$ be two fuzzy mappings. Suppose that for each $x \in X$, there exists $\alpha(x) \in(0,1]$ such that $[S x]_{\alpha(x)},[T x]_{\alpha(x)}$ are nonempty closed bounded subsets of $X$ and

$$
\begin{aligned}
H\left([S x]_{\alpha(x)},[T y]_{\alpha(y)}\right) \leq & a_{1} d\left(x,[S x]_{\alpha(x)}\right)+a_{2} d\left(y,[T y]_{\alpha(y)}\right) \\
& +a_{3}\left[d\left(x,[T y]_{\alpha(y)}\right)+d\left(y,[S x]_{\alpha(x)}\right)\right]+a_{4} d(x, y),
\end{aligned}
$$

for all $x, y \in X$, where $a_{1}, a_{2}, a_{3}, a_{4}$ are non-negative real numbers with $a_{1}+a_{2}+2 a_{3}+a_{4}<1$. Then there exists $z \in X$ such that $z \in[S z]_{\alpha(z)} \cap[T z]_{\alpha(z)}$.

Remark 2.2 Azam and Arshad [6] pointed out that the proof [5, Theorem 3.1] is incorrect and incomplete, and presented the right version of this result. In fact, by Corollary 2.2 we easily see that although there exist mistakes in the proof of Theorem 3.1 in [5], its conclusion is correct. Moreover, Corollary 2.2 also shows that Theorem 4 in [6] is not the right version of Theorem 3.1 in [5], but the special case of Theorem 3.1 in [5].

Similarly, applying Corollary 2.1 or Theorem 2.3, we can establish the following fixed point theorem for generalizing Park-Jeong-type fuzzy mappings (see [11]).

Theorem 2.4 Let $(X, d)$ be a complete metric space. Let $S, T: X \rightarrow \mathscr{F}(X)$ be two fuzzy mappings. Suppose that for each $x \in X$, there exists $\alpha(x) \in(0,1]$ such that $[S x]_{\alpha(x)},[T x]_{\alpha(x)}$ are nonempty closed bounded subsets of $X$ and

$$
H\left([S x]_{\alpha(x)},[T y]_{\alpha(y)}\right) \leq k\left[d\left(x,[S x]_{\alpha(x)}\right) \cdot d\left(y,[T y]_{\alpha(y)}\right)\right]^{\frac{1}{2}},
$$

for all $x, y \in X$, where $0<k<1$. Then there exists $z \in X$ such that $z \in[S z]_{\alpha(z)} \cap[T z]_{\alpha(z)}$.

Proof Since $0<k<1$, we can take $1-k=4 \delta>0, \eta=\delta^{2}>0$ and let $a_{1}=\frac{k}{2}+3 \delta, a_{2}=\frac{k}{2}, a_{3}=\delta$, $a_{4}=0, a_{5}=\eta=\delta^{2}$, then we have $\sum_{i=1}^{5} a_{i}=1+\eta, a_{3}+a_{4}+a_{5}=\delta+\delta^{2}<1, a_{1}+a_{4}=\frac{k}{2}+3 \delta<1$, 
$a_{2}+a_{3}=\frac{k}{2}+\delta<1$ and $\left(a_{1}-a_{2}\right)\left(a_{3}-a_{4}\right)=3 \delta^{2}>2 \delta^{2}=2 \eta$. Moreover, it is evident that

$$
\begin{aligned}
H\left([S x]_{\alpha(x)},[T y]_{\alpha(y)}\right) \leq & k\left[d\left(x,[S x]_{\alpha(x)}\right) \cdot d\left(y,[T y]_{\alpha(y)}\right)\right]^{\frac{1}{2}} \\
\leq & \frac{k}{2} d\left(x,[S x]_{\alpha(x)}\right)+\frac{k}{2} d\left(y,[T y]_{\alpha(y)}\right) \\
\leq & a_{1} d\left(x,[S x]_{\alpha(x)}\right)+a_{2} d\left(y,[T y]_{\alpha(y)}\right) \\
& +a_{3} d\left(x,[T y]_{\alpha(y)}\right)+a_{4} d\left(y,[S x]_{\alpha(x)}\right)+a_{5} d(x, y) .
\end{aligned}
$$

Then we know that the conditions of Corollary 2.1 are satisfied. Therefore, by Corollary 2.1 , the theorem is proved.

\section{Application and example}

In this section, we first establish some common fixed point theorems for Lipschitz-type multi-valued mappings in complete metric spaces. After that, we give an example to discuss the validity of the hypotheses of Theorem 2.1, by which we can claim that our results improve and extend several known results in the existing literature.

Theorem 3.1 Let $(X, d)$ be a complete metric space. Let $S, T: X \rightarrow \mathscr{C} \mathscr{B}(X)$ be two Lipschitz-type multi-valued mappings. Suppose that for each $x, y \in X$,

$$
\begin{aligned}
H(S x, T y) \leq & A_{1}(x, y) d(x, S x)+A_{2}(x, y) d(y, T y)+A_{3}(x, y) d(x, T y) \\
& +A_{4}(x, y) d(y, S x)+A_{5}(x, y) d(x, y),
\end{aligned}
$$

where $A_{1}, A_{2}, A_{3}, A_{4}, A_{5}$ are five functions from $X \times X$ to $[0,+\infty)$ such that

(i) $A_{3}(x, y)+A_{4}(x, y)+A_{5}(x, y)<1$ for all $x, y \in X$;

(ii) $\inf _{x, y \in X}\left\{1-A_{1}(x, y)-A_{4}(x, y)\right\}=a, \inf _{x, y \in X}\left\{1-A_{2}(x, y)-A_{3}(x, y)\right\}=b$,

$$
\sup _{x, y \in X}\left\{A_{1}(x, y)+A_{3}(x, y)+A_{5}(x, y)\right\}=A, \sup _{x, y \in X}\left\{A_{2}(x, y)+A_{4}(x, y)+A_{5}(x, y)\right\}=B \text {, }
$$

with $a, b, A, B>0$ and $A B<a b$. Then there exists $z \in X$ such that $z \in S z \cap T z$.

Proof Let the fuzzy mappings $S, T: X \rightarrow \mathscr{F}(X)$ be defined as $S(x)=\chi_{S(x)}$ and $T(x)=\chi_{T(x)}$, where $\chi_{A}$ is the characteristic function on any subset $A$ of $X$. Using the facts $[S x]_{\alpha(x)}=S(x)$, $[T x]_{\alpha(x)}=T(x)$ for any $\alpha(x) \in(0,1]$, it is evident that $S$ and $T$ satisfy the conditions of Theorem 2.1. Then, by Theorem 2.1, the theorem is proved.

By the proofs of Corollary 2.1 and Theorem 3.1, we can get the following theorem.

Theorem 3.2 Let $(X, d)$ be a complete metric space. Let $S, T: X \rightarrow \mathscr{C} \mathscr{B}(X)$ be two multivalued mappings. Suppose that for each $x, y \in X$,

$$
H(S x, T y) \leq a_{1} d(x, S x)+a_{2} d(y, T y)+a_{3} d(x, T y)+a_{4} d(y, S x)+a_{5} d(x, y),
$$

where $a_{1}, a_{2}, a_{3}, a_{4}$ are non-negative real numbers, $a_{5}>0$ and $\eta>0$ with $\sum_{i=1}^{5} a_{i}=1+\eta$, $a_{3}+a_{4}+a_{5}<1, a_{1}+a_{4}<1, a_{2}+a_{3}<1$ and $\left(a_{1}-a_{2}\right)\left(a_{3}-a_{4}\right)>2 \eta$. Then there exists $z \in X$ such that $z \in S z \cap T z$. 
Using the same method as in the proof of Theorem 2.3, by Theorem 3.1, it is easy to establish the following fixed point theorem for Bose-Mukherjee-type multi-valued mappings (see [16]).

Corollary 3.1 (Bose and Mukherjee [16]) Let $(X, d)$ be a complete metric space. Let $S, T$ : $X \rightarrow \mathscr{C} \mathscr{B}(X)$ be two multi-valued mappings. Suppose that for each $x, y \in X$,

$$
H(S x, T y) \leq a_{1} d(x, S x)+a_{2} d(y, T y)+a_{3} d(y, S x)+a_{4} d(x, T y)+a_{5} d(x, y),
$$

where $a_{1}, a_{2}, a_{3}, a_{4}, a_{5}$ are non-negative real numbers and $\sum_{i=1}^{5} a_{i}<1$ and $a_{1}=a_{2}$ or $a_{3}=a_{4}$. Then there exists $z \in X$ such that $z \in S z \cap T z$.

Example 1 Let $X=\{0,1,2,3, \ldots\}, d$ be a discrete metric, then $(X, d)$ is a complete metric space. Define two fuzzy mappings $S, T: X \rightarrow \mathscr{F}(X)$ as follows:

$$
\begin{aligned}
& (S x)(z)=\left\{\begin{array}{ll}
1 & \text { if } z=0, \\
0 & \text { if } z \in\{1,2,3, \ldots\},
\end{array} \text { for all } x \in X ;\right. \\
& (T 2)(z)= \begin{cases}1 & \text { if } z=1, \\
0 & \text { if } z \in\{0,2,3, \ldots\},\end{cases}
\end{aligned}
$$

and for $y \in X \backslash\{2\}$,

$$
(T y)(z)= \begin{cases}1 & \text { if } z=0 \\ 0 & \text { if } z \in\{1,2,3, \ldots\}\end{cases}
$$

Then we have

$$
[S x]_{1}=[S x]_{\alpha}=\{0\} \text { for all } x \in X \text { and } \alpha \in(0,1]
$$

and

$$
[T y]_{1}=[T y]_{\alpha}=\left\{\begin{array}{ll}
\{1\} & \text { if } y=2, \\
\{0\} & \text { if } y \in X \backslash\{2\},
\end{array} \quad \text { for all } \alpha \in(0,1]\right.
$$

Now we take $A_{1}(x, y)=\frac{1}{40}, A_{2}(x, y)=\frac{19 d(x, y)+9}{40 d(x, y)+20}, A_{3}(x, y)=\frac{1}{40}, A_{4}(x, y)=\frac{1}{2}, A_{5}(x, y)=\frac{1}{40}$ for all $x, y \in X$, then we have

$$
A_{3}(x, y)+A_{4}(x, y)+A_{5}(x, y)=\frac{11}{20}<1 \quad \text { for all } x, y \in X,
$$

and

$$
\begin{aligned}
& \inf _{x, y \in X}\left\{1-A_{1}(x, y)-A_{4}(x, y)\right\}=\frac{19}{40}>0, \\
& \inf _{x, y \in X}\left\{1-A_{2}(x, y)-A_{3}(x, y)\right\}=\frac{1}{2}>0,
\end{aligned}
$$




$$
\begin{aligned}
& \sup _{x, y \in X}\left\{A_{1}(x, y)+A_{3}(x, y)+A_{5}(x, y)\right\}=\frac{3}{40}>0, \\
& \sup _{x, y \in X}\left\{A_{2}(x, y)+A_{4}(x, y)+A_{5}(x, y)\right\}=1>0,
\end{aligned}
$$

with $\frac{3}{40} \cdot 1<\frac{9}{40}<\frac{19}{40} \cdot \frac{1}{2}$, which imply that conditions (i) and (ii) of Theorem 2.1 are satisfied.

Moreover, if $x \in X$ and $y \in X \backslash\{2\}$, then

$$
\begin{aligned}
H\left([S x]_{\alpha},[T y]_{\alpha}\right)= & \leq \leq \frac{1}{40} d\left(x,[S x]_{\alpha}\right)+\frac{19 d(x, y)+9}{40 d(x, y)+20} d\left(y,[T y]_{\alpha}\right)+\frac{1}{40} d\left(x,[T y]_{\alpha}\right) \\
& +\frac{1}{2} d\left(y,[S x]_{\alpha}\right)+\frac{1}{40} d(x, y) \quad \text { for all } \alpha \in(0,1] .
\end{aligned}
$$

If $x=0$ and $y=2$, then for all $\alpha \in(0,1]$,

$$
\begin{aligned}
H\left([S 0]_{\alpha},[T 2]_{\alpha}\right)= & H(\{0\},\{1\})=1=\frac{9}{20}+\frac{1}{40}+\frac{1}{2}+\frac{1}{40} \\
\leq & \frac{1}{40} d(0,\{0\})+\frac{19+9}{40+20} d(2,\{1\}) \\
& +\frac{1}{40} d(0,\{1\})+\frac{1}{2} d(2,\{0\})+\frac{1}{40} d(0,2) .
\end{aligned}
$$

If $x=1$ and $y=2$, then for all $\alpha \in(0,1]$,

$$
\begin{aligned}
H\left([S 1]_{\alpha},[T 2]_{\alpha}\right)= & H(\{0\},\{1\})=1=\frac{1}{40}+\frac{9}{20}+\frac{1}{2}+\frac{1}{40} \\
\leq & \frac{1}{40} d(1,\{0\})+\frac{19+9}{40+20} d(2,\{1\}) \\
& +\frac{1}{40} d(1,\{1\})+\frac{1}{2} d(2,\{0\})+\frac{1}{40} d(1,2) .
\end{aligned}
$$

If $x=2$ and $y=2$, then for all $\alpha \in(0,1]$,

$$
\begin{aligned}
H\left([S 2]_{\alpha},[T 2]_{\alpha}\right)= & H(\{0\},\{1\})=1=\frac{1}{40}+\frac{9}{20}+\frac{1}{40}+\frac{1}{2} \\
= & \frac{1}{40} d(2,\{0\})+\frac{0+9}{0+20} d(2,\{1\}) \\
& +\frac{1}{40} d(2,\{1\})+\frac{1}{2} d(2,\{0\})+\frac{1}{40} d(2,2) .
\end{aligned}
$$

If $x \in\{3,4, \ldots\}$ and $y=2$, then for all $\alpha \in(0,1]$,

$$
\begin{aligned}
H\left([S x]_{\alpha},[T 2]_{\alpha}\right)= & H(\{0\},\{1\})=1<\frac{1}{40}+\frac{9}{20}+\frac{1}{40}+\frac{1}{2}+\frac{1}{40} \\
\leq & \frac{1}{40} d(x,\{0\})+\frac{19+9}{40+20} d(2,\{1\}) \\
& +\frac{1}{40} d(x,\{1\})+\frac{1}{2} d(2,\{0\})+\frac{1}{40} d(x, 2) .
\end{aligned}
$$

Hence, the conditions of Theorem 2.1 are satisfied, and there exists $0 \in X$ such that $0 \in$ $\{0\}=[S 0]_{\alpha} \cap[T 0]_{\alpha}$ for all $\alpha \in(0,1]$. But for any non-negative real numbers $a_{1}, a_{2}, a_{3}, a_{4}$, 
$a_{5}$ with $a_{1}+a_{2}+a_{3}+a_{4}+a_{5}<1$, we have

$$
\begin{aligned}
H\left([S 2]_{\alpha},[T 2]_{\alpha}\right) & =H(\{0\},\{1\})=1>a_{1}+a_{2}+a_{3}+a_{4}+a_{5} \\
& >a_{1} d(2,\{0\})+a_{2} d(2,\{1\})+a_{3} d(2,\{1\})+a_{4} d(2,\{0\})+a_{5} d(2,2)
\end{aligned}
$$

for all $\alpha \in(0,1]$. Thus $S, T$ cannot satisfy the general contractive condition $a_{1}+a_{2}+a_{3}+$ $a_{4}+a_{5}<1$.

\section{Conclusion}

In this paper, some common fixed point theorems for Lipschitz-type fuzzy mappings and Kannan-Reich-type fuzzy mappings in complete metric spaces are obtained respectively. As applications, we establish some common fixed point theorems for Lipschitz-type multi-valued mappings in complete metric spaces. Also, we give an example to show the validity of our results, which indicates that our results improve and extend the results in $[5,6,11,13,16]$ and $[14]$.

\section{Competing interests}

The authors declare that they have no competing interests.

\section{Authors' contributions}

All authors read and approved the final manuscript.

\section{Acknowledgements}

The authors thank the referee for useful comments and suggestions for the improvement of the paper. This work was supported by the Natural Science Foundation of the Jiangsu Higher Education Institutions (Grant no. 13KJB110004) and Qing Lan Project of Jiangsu Province of China.

Received: 25 April 2013 Accepted: 20 August 2013 Published: 07 Nov 2013

\section{References}

1. Weiss, MD: Fixed points and induced fuzzy topologies for fuzzy sets. J. Math. Anal. Appl. 50, 142-150 (1975)

2. Butnariu, D: Fixed point for fuzzy mapping. Fuzzy Sets Syst. 7, 191-207 (1982)

3. Heilpern, S: Fuzzy mappings and fixed point theorems. J. Math. Anal. Appl. 83, 566-569 (1981)

4. Nadler, SB: Multi-valued contraction mappings. Pac. J. Math. 30, 369-380 (1968)

5. Vijayaraju, P, Marudai, M: Fixed point theorems for fuzzy mappings. Fuzzy Sets Syst. 135, 401-408 (2003)

6. Azam, A, Arshad, M: A note on 'Fixed point theorems of fuzzy mappings' by P. Vijayaraju and M. Marudai. Fuzzy Sets Syst. 161, 1145-1149 (2010)

7. Azam, A, Beg, I: Common fixed points of fuzzy maps. Math. Comput. Model. 49, 1331-1336 (2009)

8. Azam, A, Arshad, M, Beg, l: Fixed points of fuzzy contractive and fuzzy locally contractive maps. Chaos Solitons Fractals 42, 2836-2841 (2009)

9. Frigona, M, O'Regan, D: Fuzzy contractive maps and fuzzy fixed points. Fuzzy Sets Syst. 129, $39-45$ (2002)

10. Lee, BS, Lee, GM, Cho, SJ, Kim, DS: A common fixed point theorem for a pair of fuzzy mappings. Fuzzy Sets Syst. 98 , 133-136 (1998)

11. Park, JY, Jeong, JU: Fixed point theorem for fuzzy mappings. Fuzzy Sets Syst. 87, 111-116 (1997)

12. Som, T, Mukherjee, RN: Some fixed point theorems for fuzzy mappings. Fuzzy Sets Syst. 33, 213-219 (1989)

13. Bose, RK, Sahani, D: Fuzzy mappings and fixed point theorems. Fuzzy Sets Syst. 21, 53-58 (1987)

14. Bose, RK: Some common fixed point theorems of multi-valued mappings and fuzzy mappings in ordered metric spaces. Int. J. Pure Appl. Math. 75(2), 195-211 (2012)

15. Zhang, $X$ : Common fixed point theorem of Lipschitz type mappings on cone metric space. Acta Math. Sin. New Ser. 53, 1139-1148 (2010) (in China)

16. Bose, RK, Mukherjee, RN: Common fixed points of some multivalued mappings. Tamkang J. Math. 8(2), 245-249 (1977)

17. Nguyen, HT: A note on extension principle for fuzzy sets. J. Math. Anal. Appl. 64, 369-380 (1978)

18. Reich, S: Kannan's fixed point theorem. Boll. Unione Mat. Ital. 4, 1-11 (1971)

19. Reich, S: Some remarks concerning contraction mappings. Can. Math. Bull. 14, 121-124 (1971)

20. Reich, S: Fixed points of contractive functions. Boll. Unione Mat. Ital. 5, 26-42 (1972)

21. Azam, A, Beg, I: Common fuzzy fixed points for fuzzy mappings. Fixed Point Theory Appl. 2013, 14 (2013)

10.1186/1687-1812-2013-262

Cite this article as: Song and Zhang: Common fixed point theorems for Lipschitz-type fuzzy mappings in metric spaces. Fixed Point Theory and Applications 2013, 2013:262 